Mark McClure, Department of Mathematics, UNC-Asheville

Asheville, NC 28801, e-mail:mcmcclur@bulldog.unca.edu

\title{
THE PREVALENT DIMENSION OF GRAPHS
}

\begin{abstract}
We show that the set of functions in $C[0,1]$ with a graph of packing dimension 2 (or, equivalently, upper entropy dimension 2 ) is prevalent.
\end{abstract}

\section{Prevalence}

In his excellent monograph Measure and Category [8], Oxtoby compares and contrasts the most familiar two notions of "almost nowhere" on the real line. The extension of these ideas, Lebesgue measure zero and Baire first category, to infinite dimensional spaces is an interesting problem.

The notions of Baire category extend immediately to any complete, separable metric space and, in particular, to $C[0,1]$. A set is said to be of first category or meager if it may be expressed as a countable union of nowhere

dense sets. A set is said to be generic or comeager if it is the complement of a meager set. A classic theorem of Banach states that the set of functions in $C[0,1]$ which are nowhere differentiable forms a comeager subset ([8] chapter 11). This is frequently phrased as, the generic continuous function is nowhere differentiable. As another example, Humke and Petruska [4] prove that the set of functions in $C[0,1]$ whose graph has lower entropy index one is comeager and the set of functions in $C[0,1]$ whose graph has upper entropy dimension two is comeager. See section 2 for definitions. Their statement that the generic function in $C[0,1]$ has a graph with lower entropy index 1 strengthens a theorem of Mauldin and Williams which states that the generic function in $C[0,1]$ has a graph with Hausdorff dimension 1 ([7] Theorem 2).

There are fundamental difficulties, however, with attempts to extend measures to infinite dimensional spaces. Prevalence is a notion defined in [5] which generalizes the measure theoretic "almost nowhere" without actually defining a measure on the entire space. An equivalent notion was originally introduced

Key Words: Prevalence, Fractal Dimensions

Mathematical Reviews subject classification: 26A27,28A80,28C20,54E52

Received by the editors June 3, 1996 
in [1] as pointed out in [6]. Prevalence is defined as follows: Let $V$ be a Banach space. A Borel set $A \subset V$ will be called shy if there is a positive Borel measure $\mu$ on $V$ such that $\mu(A+v)=0$ for every $v \in V$. More generally, a subset of a shy Borel set will be called shy. In [5] it is shown that shyness satisfies all the properties one would expect of a generalization of measure zero. For example:

1. Shyness is shift invariant.

2. Shyness is closed under countable unions.

3. A subset of a shy set is shy.

4. A shy set has empty interior.

5. If $V=\mathbb{R}^{n}$, then the shy sets coincide with the measure zero sets.

The complement of a shy set will be called prevalent. The purpose of this paper is to present a result similar to Humke and Petruska's, but phrased in terms of the measure theoretic notion of prevalence.

\section{Dimension}

In this section, we define the upper entropy index, $\Delta$, and from that the upper entropy dimension, $\widehat{\Delta}$. These notions are equivalent to the well known packing index and packing dimension of Taylor and Tricot [9]. Many readers will, also, recognize these definitions as the upper box counting dimension and the modified upper box counting dimension in [3] sections 3.1 through 3.3. Our notation follows [2] section 6.5. Proofs of the equivalences of the various definitions may be found in [3] or [10].

For $\varepsilon>0$, the $\varepsilon$-square mesh for $\mathbb{R}^{2}$ is defined as the collection of closed squares $\{[i \varepsilon,(i+1) \varepsilon] \times[j \varepsilon,(j+1) \varepsilon]\}_{i, j \in \mathbf{Z}}$. For a totally bounded set $E \subset \mathbb{R}^{2}$, define

$$
N_{\varepsilon}(E)=\# \text { of } \varepsilon \text {-mesh squares which meet } E
$$

and

$$
\Delta(E)=\limsup _{\varepsilon \rightarrow 0} \frac{\log N_{\varepsilon}(E)}{-\log \varepsilon} .
$$

An easy but important property of $\Delta$ is that it respects closure. That is $\Delta(E)=\Delta(\bar{E})$. Another $([\mathrm{F}]$ p. 41) is that the limsup need only be taken along any sequence $\left\{c^{n}\right\}_{n=1}^{\infty}$ where $c \in(0,1)$ and we still obtain the same value. One problem with $\Delta$ is that it is not $\sigma$-stable. In other words it is possible that $\Delta\left(\cup_{n} E_{n}\right)>\sup _{n}\left\{\Delta\left(E_{n}\right)\right\}$. For example, $\Delta(\mathbb{Q})=1$ even though 
Q is countable. For this reason, $\Delta$ is used to define a new set function, $\widehat{\Delta}$, defined by:

$$
\widehat{\Delta}(E)=\inf \left\{\sup _{n}\left\{\Delta\left(E_{n}\right)\right\}: E=\cup_{n} E_{n}\right\}
$$

This new $\sigma$-stable set function, $\widehat{\Delta}$, is the upper entropy dimension.

We could, also, define a lower entropy index, and from that a lower entropy dimension, by replacing the limsup in equation 1 by a liminf. We will not refer to the lower entropy dimension any further, so we will not develop the notation here.

We may now state the main result. Let $C[0,1]$ denote the Banach space of continuous, real valued functions defined on $[0,1]$ with the uniform metric $\rho$. For $f \in C[0,1]$, let $G(f)=\{(x, f(x)): x \in[0,1]\}$ denote the graph of $f$.

Theorem 2.1. The set $\{f \in C[0,1]: \widehat{\Delta}(G(f))=2\}$ is a prevalent subset of $C[0,1]$.

\section{Application}

In this section, we prove several lemmas and Theorem 2.1. First we fix some notation. Let $I=\left[k 2^{-m},(k+1) 2^{-m}\right] \subset[0,1]$ be a dyadic interval, where $k, m \in \mathbb{N}$ are fixed. For $f \in C[0,1]$, let $G_{I}(f)=\{(x, f(x))\}_{x \in I}$ be that portion of the graph of $f$ lying over $I$. For any interval $[a, b] \subset[0,1]$ define $R_{f}[a, b]=\sup \{|f(x)-f(y)|: a<x, y<b\}$. For $n>m$, let

$$
M_{2^{-n}}(f)=2^{n} \sum_{i=k 2^{n-m}}^{(k+1) 2^{n-m}-1} R_{f}\left[i 2^{-n},(i+1) 2^{-n}\right]
$$

For $\gamma \in[1,2)$, let $A_{\gamma}=\left\{f \in C[0,1]: \Delta\left(G_{I}(f)\right)>\gamma\right\}$.

Lemma 3.1. For every $f \in C[0,1]$ and natural number $n>m$,

$$
M_{2^{-n}}(f) \leq N_{2^{-n}}\left(G_{I}(f)\right) \leq 2^{n-m+1}+M_{2^{-n}}(f) .
$$

Proof. See [3] proposition 11.1.

Corollary 3.1. For every non-constant $f \in C[0,1]$,

$$
\Delta\left(G_{I}(f)\right)=\limsup _{n \rightarrow \infty} \frac{\log M_{2^{-n}}(f)}{\log 2^{n}} .
$$


Proof. Note that $\liminf _{n \rightarrow \infty} 2^{-n} M_{2^{-n}}(f)>0$. Thus, there is a positive, finite bound $T$ so that

$$
1 \leq \frac{N_{2^{-n}}\left(G_{I}(f)\right)}{M_{2^{-n}}(f)} \leq \frac{2^{n-m-1}+M_{2^{-n}}(f)}{M_{2^{-n}}(f)} \leq T .
$$

The result easily follows.

Lemma 3.2. The set $\left\{f \in C[0,1]: \Delta\left(G_{I}(f)\right)=2\right\}$ is a $G_{\delta}$ subset of $C[0,1]$ and each set $A_{\gamma}$ is a $G_{\delta \sigma}$.

Proof. For any rational number $q \in(1,2)$ and any natural number $n>m$, let

$$
A_{q}(n)=\left\{f \in C[0,1]: \frac{\log M_{2^{-n}}(f)}{\log 2^{n}}>q\right\} .
$$

Note that each $A_{q}(n)$ is open, as $M_{2^{-n}}(f)$ varies continuously with $f$. Now

$$
A_{\gamma}=\bigcup_{q \in \mathbb{Q} \cap(\gamma, 2)} \bigcap_{j=1}^{\infty} \bigcup_{n=j}^{\infty} A_{q}(n)
$$

and

$$
\left\{f \in C[0,1]: \Delta\left(G_{I}(f)\right)=2\right\}=\bigcap_{q \in \mathbb{Q} \cap(1,2)} \bigcap_{j=1}^{\infty} \bigcup_{n=j}^{\infty} A_{q}(n),
$$

which expresses the sets in the desired manner. $\square$

Lemma 3.3. For all $f \in C[0,1]$ and $\lambda \neq 0, \Delta\left(G_{I}(f)\right)=\Delta\left(G_{I}(\lambda f)\right)$.

Proof. This is a simple consequence of the fact that $R_{\lambda f}[a, b]=\lambda R_{f}[a, b] . \square$.

Lemma 3.4. For all $f, g \in C[0,1]$,

$$
\Delta\left(G_{I}(f+g)\right) \leq \max \left\{\Delta\left(G_{I}(f)\right), \Delta\left(G_{I}(g)\right)\right\} .
$$

Proof. This is a simple consequence of the inequality

$$
R_{f+g}[a, b] \leq R_{f}[a, b]+R_{g}[a, b] \leq 2 \max \left\{R_{f}[a, b], R_{g}[a, b]\right\} \cdot \square
$$

Lemma 3.5. For all $\gamma<2, A_{\gamma}$ is a prevalent, Borel set.

Proof. $A_{\gamma}$ is a Borel set by lemma 3.2. We need to show that the complement, denoted $A_{\gamma}^{c}$, is a shy set. Let $g \in C[0,1]$ satisfy $\Delta\left(G_{I}(g)\right)>\gamma$. The existence of such a $g$ is guaranteed by the fact that the generic $g \in C[0,1]$ satisfies $\Delta\left(G_{I}(g)\right)=2$ (see [4], Proposition 2). Let $\mu$ be the Lebesgue type measure 
concentrated on the line $[g]$ defined by $[g]=\{\lambda g \in C[0,1]: \lambda \in[0,1]\}$. Let $h \in C[0,1]$. We will show that $\#\left\{\left(A_{\gamma}^{c}+h\right) \cap[g]\right\}=1$. Therefore, $\mu\left(A_{\gamma}^{c}+h\right)=0$. Suppose that $f_{1}, f_{2} \in A_{\gamma}^{c}$ are such that $f_{1}+h \in[g]$ and $f_{2}+h \in[g]$. Then there exists $\lambda_{1}, \lambda_{2} \in[0,1]$ such that $f_{1}+h=\lambda_{1} g$ and $f_{2}+h=\lambda_{2} g$. This implies $h=\lambda_{1} g-f_{1}=\lambda_{2} g-f_{2}$. Thus $f_{1}-f_{2}=\left(\lambda_{1}-\lambda_{2}\right) g$. This can only happen if $\lambda_{1}=\lambda_{2}$ by lemmas 3.3 and 3.4. Therefore, $f_{1}=f_{2}$. Since $h$ is arbitrary, this says that $A_{\gamma}^{c}$ is a shy set or $A_{\gamma}$ is a prevalent set. $\square$

By expressing $\left\{f \in C[0,1]: \Delta\left(G_{I}(f)\right)=2\right\}$ as a countable intersection

$$
\left\{f \in C[0,1]: \Delta\left(G_{I}(f)\right)=2\right\}=\bigcap_{\gamma \in \mathbb{Q} \cap(1,2)} A_{\gamma},
$$

we obtain the following:

Corollary 3.2. The set $\left\{f \in C[0,1]: \Delta\left(G_{I}(f)\right)=2\right\}$ is a prevalent, Borel subset of $C[0,1]$.

Finally, we prove theorem 2.1.

ProOF. Let $\left\{I_{n}\right\}_{n=1}^{\infty}$ be an enumeration of the dyadic intervals and let

$$
A_{n}=\left\{f \in C[0,1]: \Delta\left(G_{I_{n}}(f)\right)=2\right\} .
$$

Then $A_{n}$ is a prevalent, Borel set by corollary 3.2 , as is $A=\cap_{1}^{\infty} A_{n}$, being the countable intersection of prevalent, Borel sets. If

$$
B=\{f \in C[0,1]: \widehat{\Delta}(G(f))=2\},
$$

then we claim that $A \subset B$. Let $f \in A$ and let $G(f)=\bigcup_{1}^{\infty} E_{n}$ be a decomposition. Since $\Delta$ respects closure, we may assume that the $E_{n}$ 's are closed. Since $G(f)$ is closed, one of the $E_{n}$ 's must be somewhere dense by the Baire category theorem. Therefore, $E_{n} \supset G_{I_{k}}(f)$ for some $n, k$. Thus, $\Delta\left(E_{n}\right) \geq \Delta\left(G_{I_{k}}(f)\right)=2$ and $\widehat{\Delta}(G(f))=2$. Therefore, $B$ is a prevalent set since it is the superset of a prevalent, Borel set. $\square$

\section{Acknowledgments}

This paper has been greatly improved due to the comments of an anonymous referee.

\section{References}

[1] J. P. R. Christensen, "On Sets of Haar Measure Zero in Abelian Polish Groups", Israel J. Math. 13 (1972), 255-60. 
[2] G. A. Edgar. Measure, Topology, and Fractal Geometry. Springer-Verlag, New York, NY, 1990.

[3] K. J. Falconer. Fractal Geometry: Mathematical Foundations and Applications. John Wiley and Sons, West Sussex, England, 1990.

[4] P. D. Humke and G. Petruska. The Packing Dimension of a Typical Continuous Function is 2. Real Analysis Exchange, 14 (1989), 345-58.

[5] Brian Hunt, Tim Sauer, and James Yorke. Prevalence: A Translation Invariant "Almost Every" on Infinite Dimensional Spaces, Bulletin of the American Mathematical Society, 27 (1992), 217 - 38.

[6] Brian Hunt, Tim Sauer, and James Yorke. "Prevalence: An Addendum", Bulletin of the American Mathematical Society, 28 (1993), 306-7.

[7] R. Daniel Mauldin and S. C. Williams. "On the Hausdorff Dimension of Some Graphs", Transactions of the American Mathematical Society, 298 (1986), 793-803.

[8] John C. Oxtoby. Measure and Category (2nd ed.). Springer-Verlag, New York, NY, (1980).

[9] S. J. Taylor and C. Tricot, "Packing Measure and its Evaluation for a Brownian Path." Trans. Amer. Math. Soc. 288 (1985), 679-699.

[10] C. Tricot, "Two Definitions of Fractional Dimension." Math. Proc. Camb. Phil. Soc. 91 (1982), 52-74. 